\title{
Nanoscale chemical composition analysis using peptides targeting inorganic materials (Supporting information)
}

\author{
Yuki Arai ${ }^{1}$, Ken-Ichiro Okabe ${ }^{1}$, Hiroshi Sekiguchi ${ }^{2}$, Tomohiro Hayashi ${ }^{1,3,}{ }^{*}$, and Masahiko Hara ${ }^{1,3}$ \\ ${ }^{1}$ Department of Electronic Chemistry, Interdisciplinary Graduate School of Science and Engineering, \\ Tokyo Institute of Technology, \\ 4259 Nagatsuta-cho, Midori-ku, Yokohama, Kanagawa 226-8502, Japan \\ ${ }^{2}$ Graduate School of Frontier Sciences, The University of Tokyo \\ 5-1-5 Kashiwanoha, Kashiwa City, Chiba, 277-8561, Japan \\ ${ }^{3}$ Flucto-Order Functions Research Team, Advanced Science Institute, RIKEN, 2-1 Hirosawa, Wako, \\ Saitama 351-0198, Japan \\ * corresponding author \\ e-mail:hayashi@echem.titech.ac.jp
}

\section{Contents:}

1. List of chemicals used in this work

2. Preparation of the AFM probes modified with TBP

3. Filtering of force curves

4. A histogram of adhesion force obtained with the Type II probe

5. Chemical composition images at a different location

6. Loading rate dependence of single-molecule adhesion force between TBP and Ti 


\section{List of chemicals used in this work}

Ethanol (purity: 99.8\%; Wako Pure Chemical Industries, Osaka, Japan), dehydrated toluene (Kanto Chemical Co., Tokyo, Japan), 2-aminoethanethiol (Wako), N-hydroxysuccinimide-PEG24 maleimide ester (NHS-PEG-MAL, 95\%; Quanta Biodesign, Powell, OH), m-dPEG24-NHS (NHS-PEG-OMe, 98\%; Quanta Biodesign), polyoxyethylene sorbitan monolaurate (Tween20, Wako) and sodium chloride (Wako), Ti-binding peptide (RKLPDAPGMHTWC, purity 95\%, Biotech Lab, Japan), which have a cystaine at the C-terminus, were used without further purification. Milli-Q water $(\sim 18.7 \mathrm{M} \Omega \cdot \mathrm{cm}$; Millipore, Billerica, MA) was used in this experiment. Silica microspheres of $4 \mu \mathrm{m}$ (Polysciences, Warrington, PA) were used after washed several times with water and ethanol by decantation.

\section{Preparation of the AFM probes modified with TBP}

Details of the modification of the AFM probes (Type I to III) are summarized in Figs. S.2. As to the Type I probe, TBP was directly immobilized on the probe surface via a gold-sulfur bond [Fig. S.2(a)]. The Type II and III was prepared by step-by-step by using NHS- and maleimide-mediated conjugations. (Figs. S.2(b) and (c)) 


\section{Type I probe}

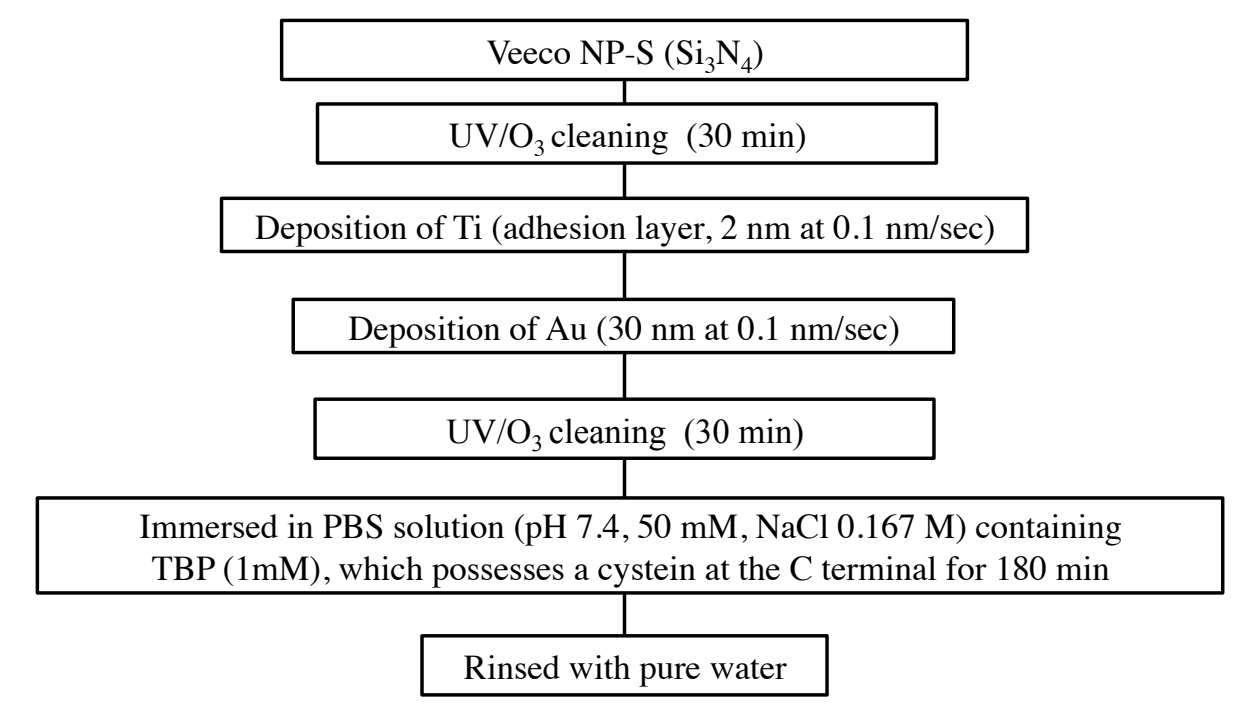

Figure S.2(a)

\section{Type II probe}

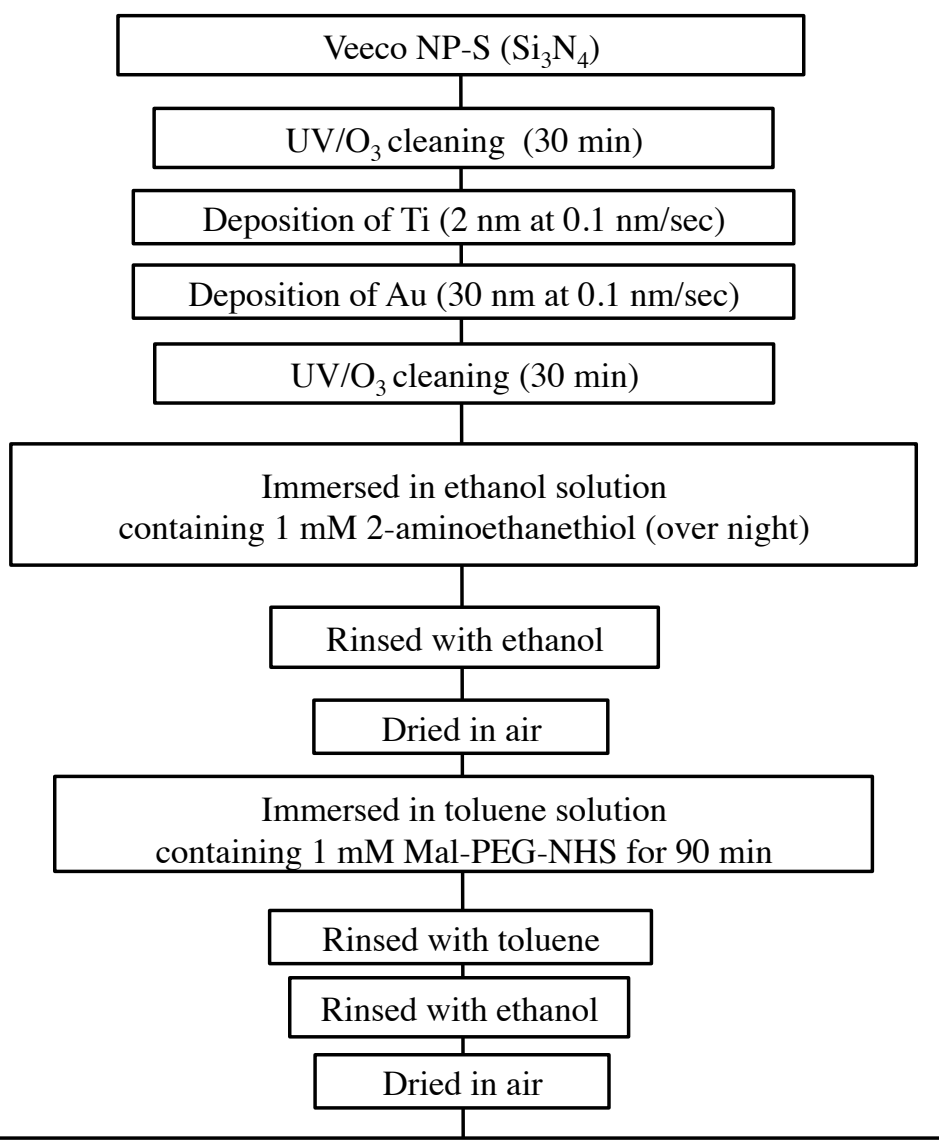

Immersed in PBS solution (pH 7.4, 50 mM, $\mathrm{NaCl} 0.167 \mathrm{M}$ ) containing TBP $(1 \mathrm{mM})$, which possesses a cystein at the $\mathrm{C}$ terminal for $45 \mathrm{~min}$

Rinsed with water 
Figure S.2(b)

\section{Type III probe}

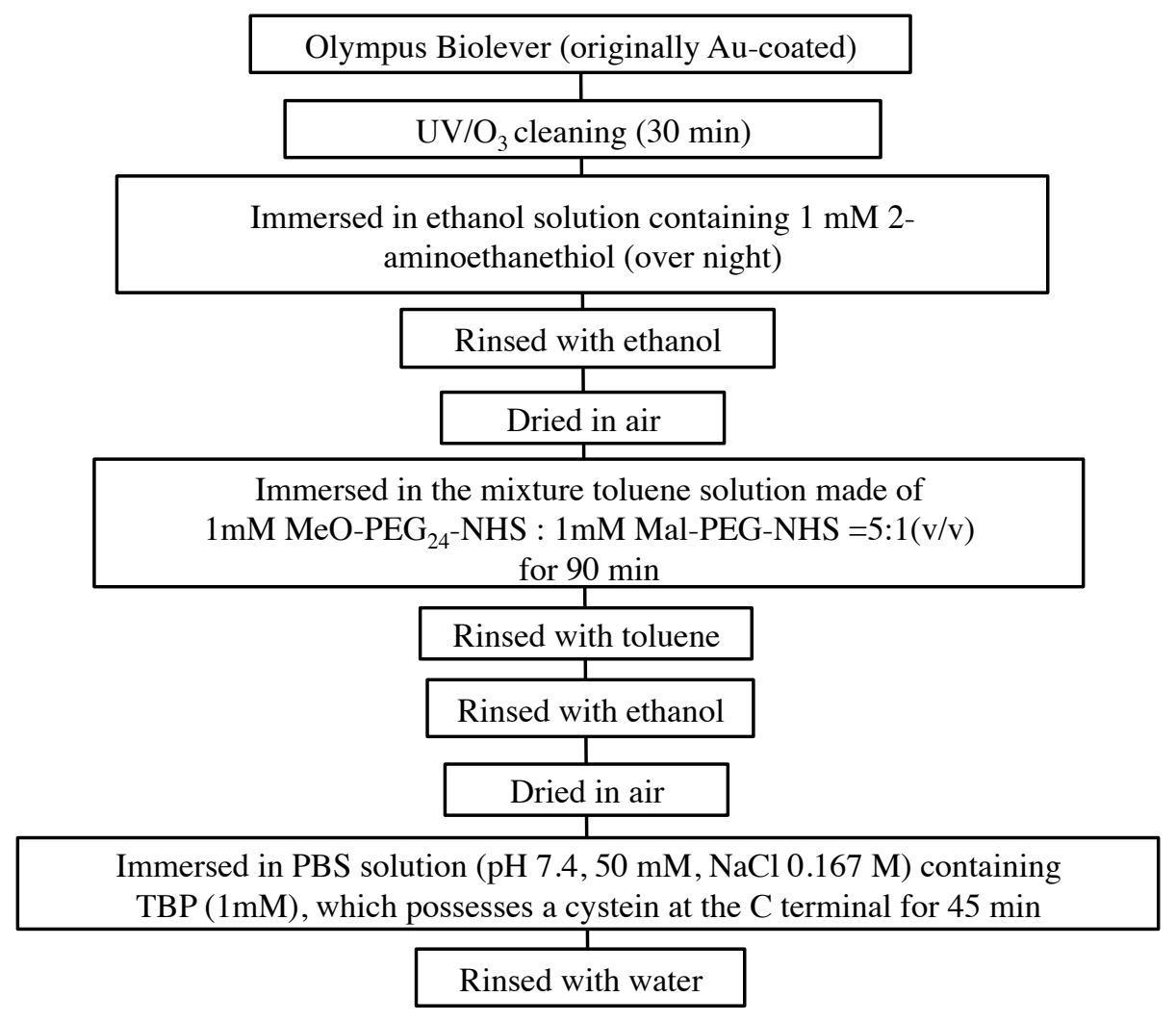

Figure S.2(c)

\section{Filtering of force curves}

We used the following criterion to pick up the adhesion event in the force-distance curves. 1.

Force zero was defined by averaging the last ten points that are farthest from the surface. 2 .

Differences in deflections between two nearest points were averaged to estimate the amplitude of the thermal motion of the cantilever. 3. If the dip in the force-distance curve is greater than twice of the 
thermal motion, it was counted as the adhesion event. We also employed the filtering method proposed by Kasas et al., ${ }^{1}$ and found that both approaches provided qualitatively same results.

\section{A histogram of adhesion force obtained with the Type II probe}

A histogram of the adhesion forces originated within the contact between Ti and Type II

probe is presented in Fig. S.4. Due to high density of TBP on the probe surface, the histogram shows only one broad peak. When we decreased the density (Type III probe), "quantized" peaks were observed (Figs. 7 in the main manuscript)

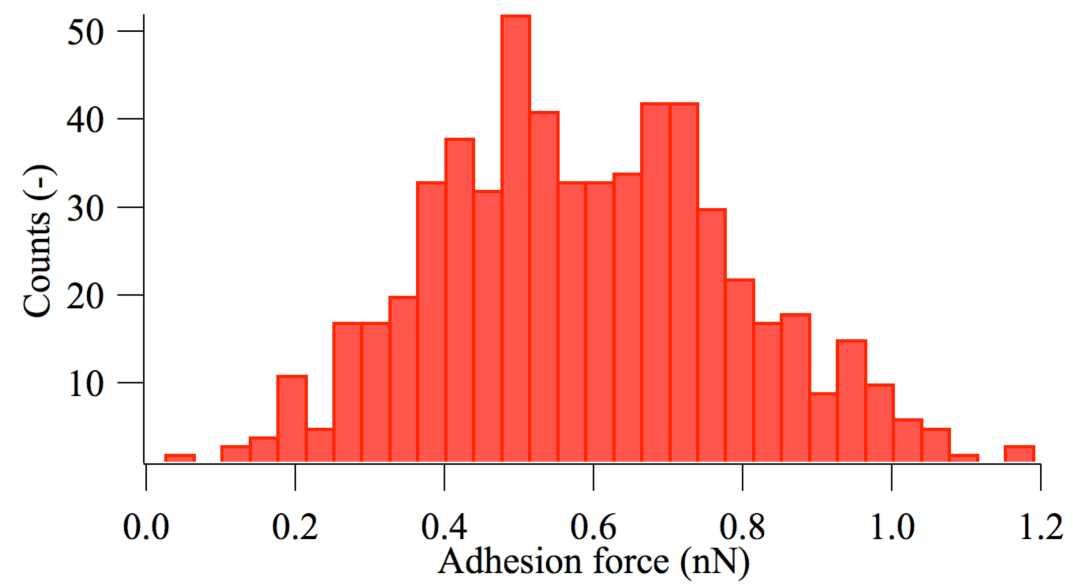

Figure S. 4. A histogram of the adhesion forces originated within the contact between Ti and Type II probe at $\tau_{\mathrm{c}}=1$ sec. 


\section{Chemical composition images at a different location}

Reproducibility of the force mapping was performed with a different Type II probe, which was prepared with the same procedure. The high reproducibility of the chemical composition imaging was confirmed.

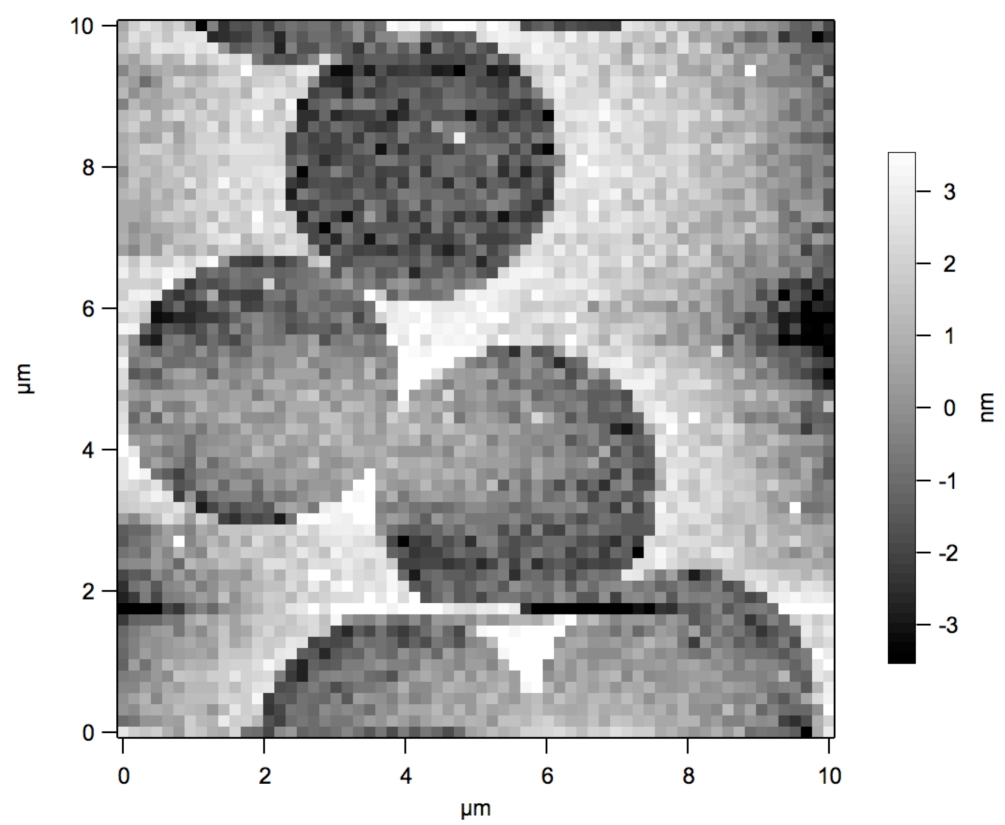

(a)

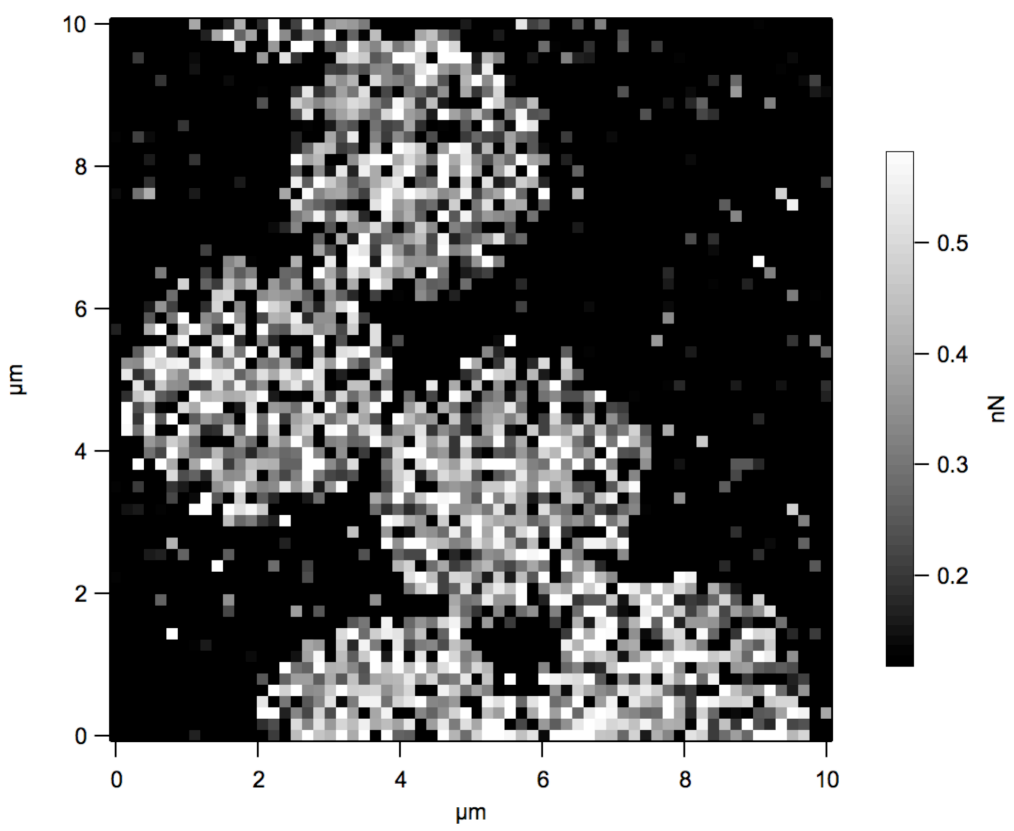

(b) 
Figure S.5 (a) Topographic and (b) adhesion force images $(10 \times 10 \mu \mathrm{m})$ of the patterned Ti-Au surfaces obtained by the Type II probe $\left(\tau_{\mathrm{c}}=1 \mathrm{sec}\right)$

\section{Loading rate dependence of single-molecule adhesion force between TBP and Ti}

In figure S.6 we show the histograms of the adhesion forces generated from several hundreds of force curves at each loading rate. The histograms were fitted by multiple Gaussian functions, and the top of the value in the weakest peak were assigned as a most probable adhesion force of single molecule, $f^{*}$.

a

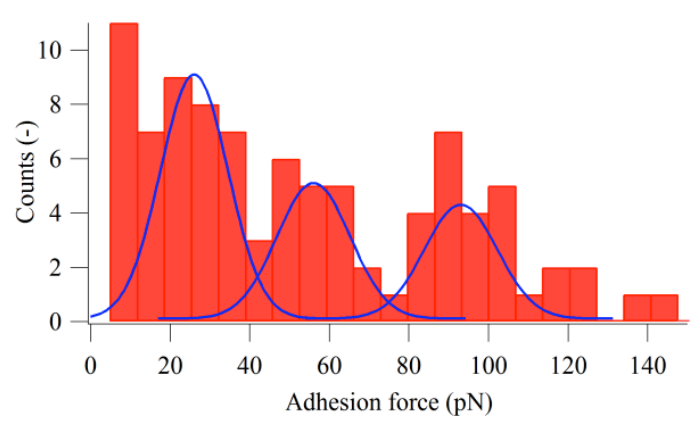

c

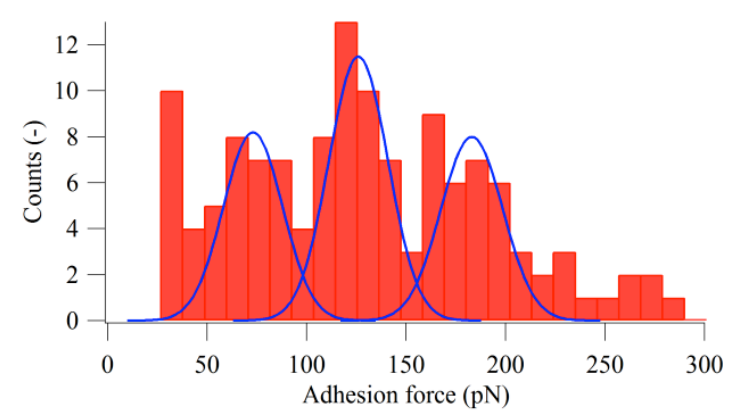

e b

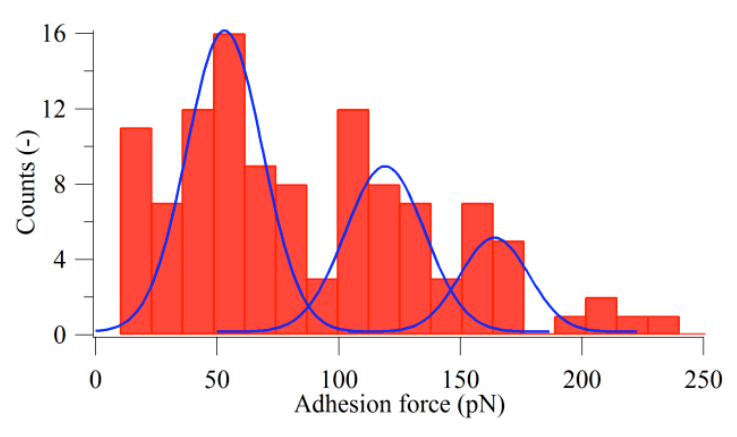

d

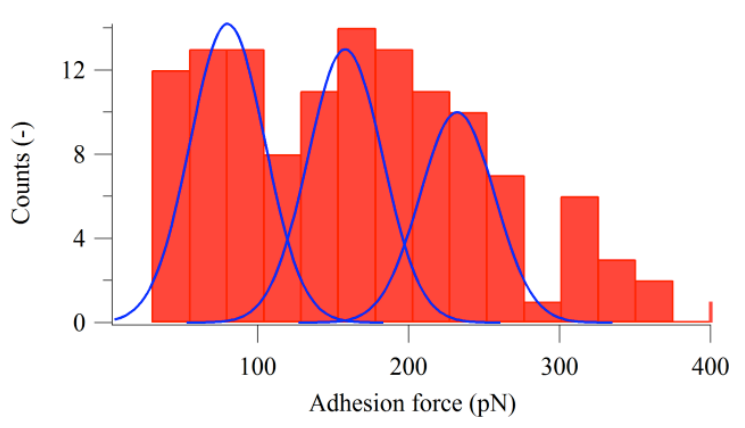

f 

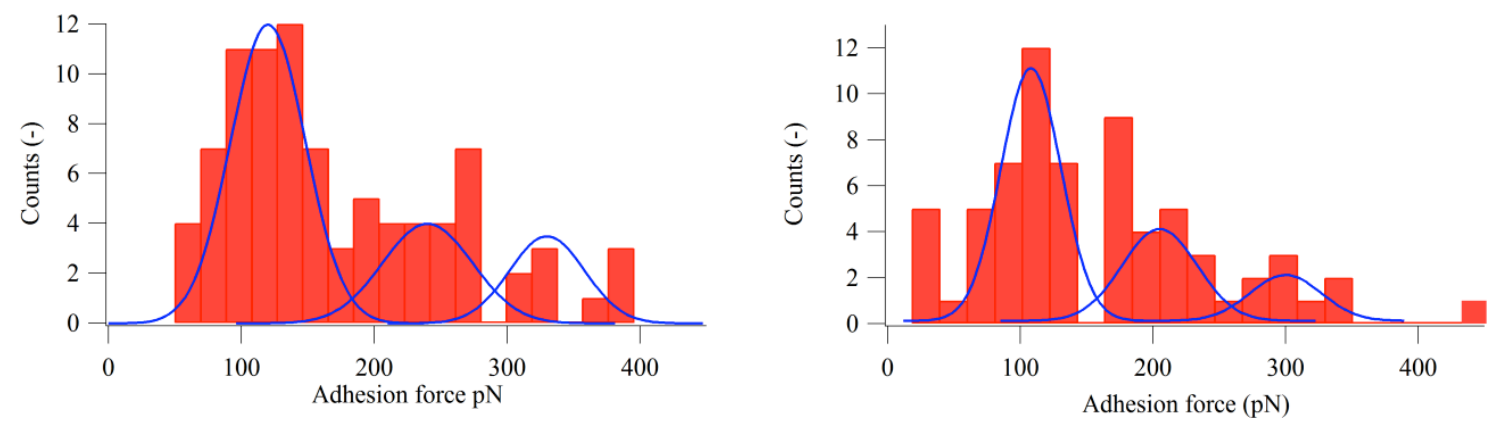

Figure S. 6. The histograms of adhesion forces at each loading rate. (a) loading rate, $v_{f}: 170 \pm 29.6 \mathrm{pN} / \mathrm{sec}$, the most probable adhesion force of single molecule, $f^{*}: 26 \mathrm{pN}$; (b) $408.8 \pm 36.5 \mathrm{pN} / \mathrm{sec}, 53 \mathrm{pN}$; (c) $1420 \pm 232 \mathrm{pN} / \mathrm{sec}, 73 \mathrm{pN}$; (d) $5925 \pm 956 \mathrm{pN} / \mathrm{sec}, 80 \mathrm{pN}$; (e) $16932 \pm 1952 \mathrm{pN} / \mathrm{sec}, 120 \mathrm{pN}$; (f) $52303 \pm 8876 \mathrm{pN} / \mathrm{sec}, 106 \mathrm{pN}$.

\section{References:}

1. $\quad$ Kasas, S.; Riederer, B. M.; Catsicas, S.; Cappella, B.; Dietler, G., Rev. Sci. Instrum. 2000, 71 (5), 2082-2086. 